\title{
An Extension of Jensen's Theorem for the Derivative of a Polynomial and for Infrapolynomials
}

\author{
O. Shisha
}

(February 20, 1962)

\begin{abstract}
The purpose of the paper is to generalize Jensen's theorem on the zeros of the derivative of a real polynomial. Results are first established for infrapolynomials and therefrom derived for expressions of the form $G^{\prime}(z)$ and $a G(z)+b z G^{\prime}(z)$, where $G(z)$ is a (complex) polynomial and $a, b$ are (complex) constants. An implicit aim the paper tries to serve is to further show how investigation of infrapolynomials may be of help to the classical study of the geometrical relation between the zeros of a polynomial and those of its derivative (or related polynomials).
\end{abstract}

1. Let $S$ be a subset of the (open) complex plane. An infrapolynomial ${ }^{1}$ on $S$ is a polynomial $A(z) \equiv z^{n}+a_{n-1} z^{n-1}+\ldots .+a_{0}(n \geq 1)$ having the property: there does not exist a polynomial $B(z) \equiv z^{n}+b_{n-1} z^{n-1}+\ldots .+b_{0}(\not \equiv A(z))$ such that

$$
\begin{gathered}
|B(z)|<|A(z)| \text { whenever } z \epsilon S \text { and } A(z) \neq 0, \\
B(z)=0 \text { whenever } z \epsilon S \text { and } A(z)=0 .
\end{gathered}
$$

For example [4], if $S$ is infinite, closed and bounded, then for $n=1,2, \ldots$ the 'Tchebycheff polynomial ${ }^{2}$ of degree $n$ for $S$ is an infrapolynomial on $S$.

2 . Another example of an infrapolynomial is the following $[10$, sec. 1 , and 2 , sec. 10 , p. 100 . Cf. also 4 , sec. 5]. Let $G(z)$ be a nonconstant polynomial, $\zeta_{1}$, $\zeta_{2}, \ldots, \zeta_{m}$ its distinct zeros $\left(\right.$ say $\left.G(z) \equiv \alpha \Pi_{\nu=1}^{m}\left(z-\zeta_{\nu}\right)^{p_{\nu}}\right)$, and assume that $m \geq 2$. Then

$$
A(z) \equiv\left(\sum_{\nu=1}^{m} p_{v}\right)^{-1} \frac{G^{\prime}(z)}{G(z)} \Pi_{\nu=1}^{m}\left(z-\zeta_{\nu}\right)
$$

is an infrapolynomial on $\left\{\zeta_{1}, \zeta_{2}, \ldots, \zeta_{m}\right\}$.

3. Various results have been obtained concerning the location of the zeros of infrapolynomials. The first of these was given (implicitly) by L. Fejér [1] and others were proved by M. Fekete and J. von Neumann [4], J. L. Walsh [27], J. L. Walsh and T. S. Motzkin [31, 11, 12, 13, 14], M. Fekete [2], M. Marden [9], and O. Shisha $[16,17]$. One of the most typical theorems in this direction is the following (a special case of [5], Theorem X and the end of sec. 10, p. 66 . Cf. also [4], Theorem 1). Let $\xi$ and $\eta$ be distinct zeros of an infrapolynomial on a closed and bounded set $S$. Let $L$ be the perpendicular bisector of the segment

\footnotetext{
1 Originally called "extremal polynomial." This concept was introduced by M. Fekete and J. von Neumann [4]. The term "infrapolynomial" is due to Professors T. S. Motzkin and J. L. Walsh [12]. Figures in brackets indicate the literature references at the end of this paper.

2 This is the unique polynomial $T(z)$ of the form $\left(^{*}\right) z^{n}+c_{n-1} z^{n-1}+\ldots+c_{0}$ such that the max $[|T(z)|, z$ on $S] \leq \max [|P(z)|, z$ on $S]$ for every polynomial $P(z)$ of the form $\left({ }^{*}\right)$.
}

$(\xi, \eta)$. For every $z$, let $C_{z}$ denote the closed disk having the segment joining $z$ to its mirror image in $L$ as a diameter $\left(C_{z}=\{z\}\right.$ if $\left.z \epsilon L\right)$, and let $C_{z}^{\prime}$ be the closure of the complement of $C_{z}$. Then there exists a point $p$ of $S$ such that $\xi$ and $\eta$ belong to $C_{p}$. There exists also a point $q$ of $S$ such that $\xi$ and $\eta$ belong to $C_{q}^{\prime}$.

4. In Theorem 1 we generalize the last result by substituting in the latter a circle for the line $L$.

Theorem 1: Let $\xi$ and $\eta$ be distinct zeros of $A(z)$, an infrapolynomial on a closed and bounded set $S$. Let $K$ be a circle whose center $\zeta$ does not belong to $S$, such that $\xi$ and $\eta$ are symmetric (i.e., inverse) to each other with respect to $K$. For every $z(\neq \zeta)$ let $C_{z}$ denote the closed disk having the segment joining $z$ to its inverse with respect to $K$ as a diameter $\left(C_{z}=\{z\}\right.$ if $z \epsilon K)$, and let $C_{2}^{\prime}$ be the closure of the complement of $C_{z}$. Then there exists a point $p$ of $S$ such that $\xi$ and $\eta$ belong to $C_{p}$. There exists also a point $q$ of $S$ such that $\xi$ and $\eta$ belong to $C_{q}^{\prime}$.

5. To prove this theorem, we establish first the following

Lemma: Let $K:|z-\zeta|=r$ be a circle and $z_{0}, \xi_{1}, \xi_{2}$, $\eta_{1}, \eta_{2}$ points such that $z_{0} \neq \zeta, 0<\left|\xi_{i}-\zeta\right|<r, \eta_{i}$ is symmetric to $\xi_{i}$ with respect to $K(i=1,2)$ and $\xi_{2}$ lies on the (open) segment $\left(\xi_{1}, \eta_{1}\right)$. Then (using notations of Theorem 1):

(a) If $\xi_{2} \epsilon C_{z_{0}}^{\prime}$, then

$$
\left|z_{0}-\xi_{2}\right|\left|z_{0}-\eta_{2}\right|<\left|z_{0}-\xi_{1}\right|\left|z_{0}-\eta_{1}\right| .
$$

(b) If $\xi_{1} \epsilon C_{z_{0}}$ then

$$
\left|z_{0}-\xi_{2}\right|\left|z_{0}-\eta_{2}\right|>\left|z_{0}-\xi_{1}\right|\left|z_{0}-\eta_{1}\right| \text {. }
$$

Proof of the Lemma: We introduce a polar coordinate system in which the pole is $\zeta$ and the fixed ray is the one emanating from $\zeta$ and passing through $z_{0}$. Let $\left(\rho_{1}, \varphi\right),\left(\rho_{2}, \varphi\right)$ be, respectively, polar coordinates of $\xi_{1}$ and $\xi_{2}\left(0<\rho_{1}<\rho_{2}<r\right)$, and set $a=\left|z_{0}-\zeta\right|$. For every positive $x$ let

$F(x)=\left(x^{2}+a^{2}-2 a x \cos \varphi\right)\left(\left(\frac{r^{2}}{x}\right)^{2}+a^{2}-2 a \frac{r^{2}}{x} \cos \varphi\right)$. 
Then $\left(\left|z_{0}-\xi_{i}\right|\left|z_{0}-\eta_{i}\right|\right)^{2}=F\left(\rho_{i}\right)(i=1,2)$. Thus in order to prove the Lemma it suffices to show that if $\rho_{1}<t<\rho_{2}$, then $F^{\prime}(t)<0$ in case $\xi_{2} \epsilon C_{z_{0}}^{\prime}$, and $F^{\prime}(t)>0$ in case $\xi_{1} \epsilon C_{z_{0}}$.

Let $t$ be an arbitrary point of the open interval $\left(\rho_{1}, \rho_{2}\right)$ and denote by $\tau$ the point with polar coordinates $(t, \varphi)$. Set

$$
D=\left|\tau-\frac{1}{2}\left(z_{0}+z_{0}^{\prime}\right)\right|^{2}-\left|\frac{1}{2}\left(z_{0}-z_{0}^{\prime}\right)\right|^{2}
$$

where $z_{0}^{\prime}$ is the inverse of $z_{0}$ with respect to $K$. If $\xi_{2} \epsilon C_{z_{0}}^{\prime}$, then $\tau$ is an interior point of $C_{z_{0}}^{\prime}$ and $D>0$. Similarly, if $\xi_{1} \epsilon C_{z_{0}}$ then $\tau$ is an interior point of $C_{z_{0}}$ and therefore $D<0$.

We have

$$
\begin{aligned}
D=t^{2}+ & {\left[\frac{1}{2}\left(a+\frac{r^{2}}{a}\right)\right]^{2}-t\left(a+\frac{r^{2}}{a}\right) \cos \varphi } \\
& -\left[\frac{1}{2}\left(a-\frac{r^{2}}{a}\right)\right]^{2}=t^{2}+r^{2}-\frac{t}{a}\left(a^{2}+r^{2}\right) \cos \varphi .
\end{aligned}
$$

From (4), a straightforward computation yields

$$
F^{\prime}(t)=2 a^{2} t^{-3}\left(t^{4}-r^{4}\right)-2 a t^{-2}\left(t^{2}-r^{2}\right)\left(a^{2}+r^{2}\right) \cos \varphi,
$$

and in view of $(6)$,

$$
F^{\prime}(t)=2 a^{2} t^{-3}\left(t^{2}-r^{2}\right) D .
$$

Thus, if $\xi_{2} \epsilon C_{z_{0}}^{\prime}$, then $F^{\prime}(t)<0$, and if $\xi_{1} \epsilon C_{z_{0}}$, then $F^{\prime}(t)>0$.

6. Proof of Theorem 1: If one of the two points $\xi, \eta$ belongs to some $C_{z}$, so does the other. Let $\xi_{1}$ be that one of $\xi, \eta$ lying within $K$, and let $\eta_{1}$ be the other one. So in order to prove the first conclusion of Theorem 1, it suffices to show that $\xi_{1} \epsilon C$, where $C=\cup C_{z}$. Suppose $\xi_{1} \notin C$. Since $C$ is closed, we can find a point $\xi_{2}$ within $K$ and on the open segment $\left(\xi_{1}, \eta_{1}\right)$ such that $\xi_{2} \notin C$. Let $\eta_{2}$ be the inverse of $\xi_{2}$ with respect to $K$. Consider the polynomial

$$
B(z) \equiv A(z)\left[\left(z-\xi_{1}\right)\left(z-\eta_{1}\right)\right]^{-1}\left(z-\xi_{2}\right)\left(z-\eta_{2}\right) .
$$

By conclusion (a) of the Lemma, for every $z_{0} \epsilon S$, (3) holds. Thus (1) and (2) hold, contradicting our hypothesis that $A(z)$ is an infrapolynomial on $S$. Similarly one derives the second conclusion of Theorem 1 from (b) of the Lemma.

7. The notion of an infrapolynomial was generalized by J. L. Walsh and M. Zedek [34] to include more general polynomials, and further work in that direction was done by J. L. Walsh [27, 28, 29], M. Fekete and J. L. Walsh $[5,6]$, M. Zedek [36, 37, 38], O. Shisha and J. L. Walsh [23], and O. Shisha $[16,17,20,21,22]$. (Other papers related to the subject are $[3,10,11,12,15,18,19,31,32,33$, 35]). A special case studied in much detail by J. L. Walsh [27] is obtained by adding to the properties of $B(z)$ in sec. 1 the equality $b_{0}=a_{0}$.
Repeating here a particular instance of a general definition, we mention the following convention. Let $n(\geq 2)$ be an integer and $S$ a set in the (open) complex plane. An $n$-th infrapolynomial on $S$ with respect to $(0, n)$ is a polynomial $A(z) \equiv$ $\sum_{\nu=0}^{n} a_{\nu} z^{\nu}$ having the property: there does not exist a polynomial $B(z) \equiv \sum_{\nu=0}^{n} b_{\nu} z^{\nu}(\not \equiv A(z)$ satisfying (1), (2) and the equalities $b_{0}=a_{0}, b_{n}=a_{n}$.

8. Corresponding to sec. 2 , we have the following result which is a special case of a more general theorem [23, Theorem 3]. Let $G(z)$ be a polynomial $(G(0) \neq 0), \zeta_{1}, \zeta_{2}, \ldots . \zeta_{m}(m \geq 2)$ its distinct zeros, and let $\mathrm{a}$ and $\mathrm{b}$ be arbitrary complex numbers. Then

$$
A(z) \equiv\left[a+b z \frac{G^{\prime}(z)}{G(z)}\right] \Pi_{\nu=1}^{m}\left(z-\zeta_{v}\right)
$$

is an $m$-th infrapolynomial on $\left\{\zeta_{1}, \zeta_{2}, \ldots, \zeta_{m}\right\}$ with respect to $(0, m)$.

9. Theorem 2: Theorem 1 remains true if "infrapolynomial" is replaced by " $n$-th $(n \geq 2)$ infrapoly. nomial $(\neq 0)$ with respect to $(0, n), "$ provided that the center $\zeta$ of $K$ is the origin.

Indeed, we can use again the proof of Theorem 1 since $B(z)$ defined there will have the same coefficient of $z^{n}$ as $A(z)$ has, and (since $\left|\xi_{2} \eta_{2}\right|=$ square of the radius of $K=\left|\xi_{1} \eta_{1}\right|$, which implies that $\xi_{2} \eta_{2}=$ $\left.\xi_{1} \eta_{1}\right)$ the constant terms of $B(z)$ and $A(z)$ will be equal.

10. As an application of theorems 1 and 2 we have the following

Theorem 3: I. Let $\xi$ and $\eta$ be distinct zeros of $G^{\prime}(z)$ where $G(z)$ is a nonconstant polynomial. Let $\xi$ and $\eta$ be symmetric with respect to a circle $K$ whose center is not a zero of $G(z)$. Then there exists a zero $p$ of $G(z)$ such that (using a notation of Theorem 1) $\xi$ and $\eta$ belong to $C_{p}$. There exists also a zero $q$ of $G(z)$ such that $\xi$ and $\eta$ belong to $C_{q}^{\prime}$. II. Let $\xi$ and $\eta$ be distinct zeros of $a G(z)+b z G^{\prime}(z)(\not \equiv 0)$ where $G(z)$ is a polynomial $(G(0) \neq 0)$ and $a$ and $b$ are complex numbers. Let $\xi$ and $\eta$ be symmetric with respect to a circle $K$. Then the conclusions of part $I$ hold, provided that the center of $K$ is the origin.

Proof: We set $G(z) \equiv \alpha \Pi_{\nu=1}^{m}\left(z-\zeta_{\nu}\right)^{p_{\nu}}$ where $\zeta_{j} \neq \zeta_{k}$ whenever $j \neq k$ and where $p_{1}, p_{2}, \ldots, p_{m}$ are positive. To prove part I we deiine $A(z)$ as in sec. 2 and observe that $m \geq 2$ since $G^{\prime}(z)$ vanishes at the distinct points $\xi$ and $\eta$. We may assume that $G(\xi) G(\eta) \neq 0$, for otherwise we can take $p=q=\xi$ or $p=q=\eta$. Thus $\xi$ and $\eta$ are zeros of $A(z)$, which by sec. 2 is an infrapolynomial on the set of zeros of $G(z)$. The desired conclusions follow now from Theorem 1 . To prove part II, we assume that $m \geq 2$, as otherwise $\xi$ or $\eta$ is $\zeta_{1}$ and one can take $p=q=\zeta_{1}$. Define now $A(z)$ by (7). As before, we may also assume that $G(\xi) G(\eta) \neq 0$. Then $\xi$ and $\eta$ are zeros of $A(z)$, which by sec. 8 is an $m$-th infrapolynomial on $\left\{\zeta_{1}, \zeta_{2}, \ldots, \zeta_{m}\right\}$ with respect to $(0, m)$. The desired conclusions follow from Theorem 2.

11. For every $z$, let $\Gamma_{z}$ and $\Gamma_{z}^{\prime}$ be, respectively, $C_{z}$ and $C_{z}^{\prime}$ of sec. 3, with $L$ taken as the real axis. Theorem 3, I generalizes the following well known theorem, enunciated without proof by J. L. W. V. 
Jensen [7] and proved by J. L. Walsh [24]. Let $\xi$ be a nonreal zero of $A^{\prime}(z)$, where $A(z)$ is a nonconstant polynomial whose coefficients are real. Then there exists a zero $p$ of $A(z)$ such that $\xi \epsilon \Gamma_{p}$. It was proved also by J. L. Walsh that there exists a zero $q$ of $A(z)$ such that $\xi \in \Gamma_{q}^{\prime}$.

12. The last results readily imply the following limiting case of Theorem 3, I. Let $\xi$ and $\eta$ be distinct zeros of $G^{\prime}(z)$, where $G(z)$ is a nonconstant polynomial. Let $L$ be the perpendicular bisector of the segment $(\xi, \eta)$. Then (using the notation of sec. 3) there exists a zero $p$ of $G(z)$ such that $\xi$ and $\eta$ belong to $C_{p}$. There exists also a zero $q$ of $G(z)$ such that $\xi$ and $\eta$ belong to $C_{q}^{\prime}$. Indeed, we may assume (using a simple transformation) that $\eta=\bar{\xi} . \quad$ Set $G(z) \equiv \alpha \Pi_{v=1}^{n}\left(z-z_{\nu}\right), \quad A(z) \equiv \Pi_{\nu=1}^{n}\left(z-z_{v}\right)(z$ $\left.-\bar{z}_{\nu}\right)$. Then $A^{\prime}(\xi)=\left(\Pi_{\nu=1}^{n}\left(z-z_{\nu}\right)\right)_{z=\xi}^{\prime} \Pi_{\nu=1}^{n}\left(\xi-\bar{z}_{\nu}\right)+$ $\Pi_{\nu=1}^{n}\left(\xi-z_{\nu}\right) \overline{\left(\Pi_{\nu=1}^{n}\left(z-z_{\nu}\right)\right)^{\prime} z_{z}} \eta=0$. The desired conclusions follow now ${ }^{3}$ from sec. 11 . These same conclusions are implied also by the results quoted in secs. 2,3 .

13. There are many complements and generalizations of Jensen's theorem due to J. L. Walsh for which the reader is referred to the literature $[24,25,26,27$, 30].

\section{References}

[1] L. Fejér, Úber die Lage der Nullstellen von Polynomen, die aus Minimumforderungen gewisser Art entspringen, Math. Ann. 85, pp. 41-48 (1922).

[2] M. Fekete, On the structure of extremal polynomials, Proc. Natl. Acad. Sci. U.S. 37, pp. 95-103 (1951).

[3] M. Fekete, On the structure of polynomials of least deviation, Bull. Research Council Israel 5A, pp. 11-19 (1955).

[4] M. Fekete and J. von Neumann, Über die Lage der Nullstellen gewisser Minimumpolynome, Jber. Deutsch. Math. Verein. 31, pp. 125-138 (1922).

[5] M. Fekete and J. L. Walsh, On restricted infrapolynomials, J. Analyse Math. 5, pp. 47-76 (1956-57).

[6] M. Fekete and J. L. Walsh, Asymptotic behavior of restricted extremal polynomials and of their zeros, Pacific J. Math. \%, pp. 1037-1064 (1957).

[7] J. L. W. V. Jensen, Recherches sur la théorie des équations, Acta Math. 36, pp. 181-195 (1913).

[8] M. Marden, The geometry of the zeros of a polynomial in a complex variable, Mathematical Surveys, Amer. Math. Soc., No. III, New York (1949).

[9] M. Marden, On the zeros of infrapolynomials for partly arbitrary point sets, Proc. Amer. Math. Soc. 10, pp. 391-394 (1959)

[10] T. S. Motzkin and J. L. Walsh, On the derivative of a polynomial and Chebychev approximation, Proc. Amer. Math. Soc. 4, pp. 76-87 (1953).

[11] T. S. Motzkin and J. L. Walsh, Least $p$ th power polynomials on a real finite point set, Trans. Amer. Math. Soc. 78, pp. 67-81 (1955).

[12] T. S. Motzkin and J. L. Walsh, The least $p$ th power polynomials on a finite point set, Trans. Amer. Math. Soc 83 pp. 371-396 (1956).

[13] T. S. Motzkin and J. L. Walsh, Underpolynomials and infrapolynomials, Illinois J. Math. 1, pp. 406-426 (1957).
[14] T. S. Motzkin and J. L. Walsh, Location of zeros of infrapolynomials, Compositio Math. 14, pp. 50-70 (1959).

[15] T. S. Motzkin and J. L. Walsh. Polynomials of best approximation on a real finite point set. I, Trans. Amer. Math. Soc. 91, pp. 231-245 (1959).

[16] O. Shisha, On the location of the zeros of infrapolynomials (abstract), Bull. Res. Council Israel FF, pp. 44-45 (1957).

[17] O. Shisha, Properties of extremal systems of points (mimeographed), Ph. D. Thesis, 1958.

[18] O. Shisha, Nearest functions (abstract), Notices Amer. Math. Soc. 5, No. 7, p. 853 (1958).

[19] O. Shisha, A characterization of certain nearest function. (abstract), Notices Amer. Math. Soc. 6, No. 2, p. 144 (1959).

[20] O. Shisha, On generalized infrapolynomials of a certain type (abstract), Notices Amer. Math. Soc. 6, No. 3, p. $283(1959)$

[21] O. Shisha, On the structure of infrapolynomials with prescribed first and last coefficients (abstract), Notices Amer. Math. Soc. 6, No. 4, p. 393 (1959).

[22] O. Shisha, A structure theorem for infrapolynomials with prescribed coefficients (abstract), Notices Amer. Math. Soc. 8, No. 1, p. 66 (1961).

[23] O. Shisha and J. L. Walsh, The zeros of infrapolynomials with some prescribed coefficients, J. Analyse Math. 9, pp. 111-160 (1961).

[24] J. L. Wash, On the location of the roots of the derivative of a polynomial, Ann. of Math. 22, pp. 128-144 (1920).

[25] J. L. Wash, The location of critical points of analytic and harmonic functions, Colloquium Publications, Amer. Math. Soc. 34, (1950).

[26] J. L. Wash, A generalization of Jensen's theorem on the zeros of the derivative of a polynomial, Amer. Math. Monthly 62, pp. 91-93 (1955).

[27] J. L. Walsh, On infrapolynomials with prescribed constant term, J. Math. Pures Appl. 37, pp. 295-316 (1958)

[28] J. L. Walsh, On extremal approximations, a chapter in "On numerical approximation" edited by R. E. Langer, The University of Wisconsin Press, Madison, pp. 209216 (1959)

[29] J. L. Walsh, On the asymptotic properties of extremal polynomials with prescribed constant term, Math. Zeit. 73, pp. 339-345 (1960).

[30] J. L. Walsh, A new generalization of Jensen's theorem on the zeros of the derivative of a polynomial, Amer. Math. Monthly 68, pp. 978-983 (1961).

[31] J. L. Walsh and T. S. Motzkin, Polynomials of best approximation on a real finite point set, Proc. Natl. Acad. Sci. U.S. 43, pp. 845-846 (1957).

[32] J. L. Walsh and T. S. Motzkin, Polynomials of best approximation on an interval, Proc. Natl. Acad. Sci. U.S. 45, pp. $1523-1528$ (1959).

[33] J. L. Walsh and T. S. Motzkin, Best approximation within a linear family on an interval, Proc. Natl. Acad. Sci. U.S. 46, pp. 1225-1233 (1960)

[34] J. L. Walsh and M. Zedek, On generalized Tchebycheff polynomials, Proc. Natl. Acad. Sci. U.S. 42, pp. 99-104 (1956).

[35] M. Zedek, Fejér's theorem on the zeros of extremal polynomials generalized. Preliminary report, Bull. Amer. Math. Soc. 61, p. 49 (1955).

[36] M. Zedek, On generalized Tchebycheff polynomials, Ph. D. thesis (1956).

[37] M. Zedek, Infra-(n,s)-polynomials on a real set. Preliminary report, Bull. Amer. Math. Soc. 63, p. 25 (1957).

[38] M. Zedek, Strong infra-(n,s)-polynomials. Preliminary report, Bull. Amer. Math. Soc. 63, p. 127 (1957). 